\title{
Editorial
}

\section{Iterative Methods for Nonlinear Equations or Systems and Their Applications 2014}

\author{
Juan R. Torregrosa, ${ }^{1}$ Ioannis K. Argyros, ${ }^{2}$ Changbum Chun, ${ }^{3}$ \\ Alicia Cordero, ${ }^{1}$ and Fazlollah Soleymani ${ }^{4}$ \\ ${ }^{1}$ Instituto de Matemáticas Multidisciplinar, Universitat Politècnica de València, 46022 Valencia, Spain \\ ${ }^{2}$ Department of Mathematics Sciences, Cameron University, Lawton, OK 73505, USA \\ ${ }^{3}$ Department of Mathematics, Sungkyunkwan University, Suwon 440-746, Republic of Korea \\ ${ }^{4}$ Department of Mathematics, Islamic Azad University, Zahedan Branch, P.O. Box 987-98138, Zahedan, Iran
}

Correspondence should be addressed to Juan R. Torregrosa; jrtorre@mat.upv.es

Received 18 September 2014; Accepted 18 September 2014; Published 9 November 2014

Copyright ( 2014 Juan R. Torregrosa et al. This is an open access article distributed under the Creative Commons Attribution License, which permits unrestricted use, distribution, and reproduction in any medium, provided the original work is properly cited.

Many problems in computational sciences and other disciplines can be brought in the form of an equation in some abstract space using mathematical modeling. The solutions of these equations can rarely be found in closed form. That is why most solution methods for such equations are usually iterative. The construction of iterative methods for finding the solutions of nonlinear equations or systems is a fascinating task in numerical analysis and applied scientific branches. Over the last years, iterative procedures have been applied in many diverse fields like economics, engineering, physics, dynamical models, and so on. The existence of extensive literature on these iterative schemes reveals that this topic is a dynamic branch of the applied mathematics with interesting and promising applications.

This special issue was opened in February of 2014 and closed in May of 2014. There were 26 submissions in total and 12 of them were accepted for publication, after strict reviews, which gave important developments in iterative methods and their applications. The guest editors of this special issue hope that the presented results could outline new ideas for futures studies.

The basic purpose of this special issue is to present the new trends in the field of iterative methods for nonlinear problems and extend their applications in mathematics and applied sciences. The authors were invited to submit original research articles to stimulate the continuing efforts in nonlinear equations and related theories. The special issue provided a forum for researchers and scientists to communicate their recent developments and to present their novel results on nonlinear problems.

The topics of the accepted 12 papers cover the area from theory to real applications. With the help of linear and nonlinear functional analysis tools, several methods and their corresponding convergence analysis have been presented for solving nonlinear problems. All of them gave numerous numerical tests and some of them real applications. We have the pleasure to present, in alphabetical order of the first author, the selected manuscripts for this special issue.

S. Artidiello et al. present in their work a family of optimal iterative methods for solving nonlinear equations, with high order of convergence. They design this class by using weight functions technique, with functions of three variables. Some numerical tests confirm the theoretical results.

The paper of J. A. Conejero et al. presents an interesting iterative algorithm for the management of an electric carrental service. This algorithm is based on flows on networks for the acceptance of bookings by a car-rental service that permits one-way reservations.

Two manuscripts are devoted to describing iterative schemes for finding multiple roots of nonlinear equations. A biparametric family of fourth-order iterative methods for approximating multiple roots of nonlinear equations is presented by Y. I. Kim and Y. H. Geum. These iterative schemes are optimal in the sense of Kung-Traub conjecture. 
They present a wide selection of test functions for comparing their methods with other known ones. Other families of third-order iterative methods for finding multiple roots of nonlinear equations are developed by R. F. Lin et al. Mild conditions are given to assure the cubic convergence and these classes include many known schemes in this area.

R. Lin et al. introduce a new modified Halley's method for solving nonlinear equations in Banach spaces. They establish convergence theorems of Newton-Kantorovich type obtaining order three for the presented scheme. For showing the applicability of their results, several numerical examples are given.

It is known that the preconditioner presented by Hadjidimos et al. in 2003 can improve the convergence rate of the classical iterative methods to solve linear systems of equations. In the work of C. Liu and C.-L. Li, they extend this preconditioner to solve linear complementarity problems whose coefficient matrix is $M$-matrix or $H$-matrix and present a multisplitting and Schwarz method. Convergence theorems and numerical experiments for showing the efficiency of the presented methods are given.

Two papers are devoted to compute generalized inverses. $\mathrm{X}$. Liu and Z. Zuo present a new iterative method, of at least fifteenth-order convergence, for computing the generalized inverse $A_{T, S}^{(2)}$ for a given matrix $A$. They also discuss how the new method could be applied for finding approximate inverses of nonsingular square matrices. Some numerical tests are presented for showing the efficiency and superiority of the shown methods. F. Soleymani et al. present a computational iterative method, with order of convergence nine, to find approximate inverses of matrices. The extension of the proposed method for computing Moore-Penrose inverse is furnished. Numerical tests are used for comparing with different existing methods and to confirm the theoretical results.

On the other hand, F. Soleymani et al. also present a class of Steffensen-type iterative schemes for solving systems of nonlinear equations. By considering a frozen Jacobian matrix, they provide a class of $m$-step methods with order of convergence $m+1$. A new method named as SteffensenSchulz scheme is also designed.

$\mathrm{X}$. Wang proposes in his paper a wavelet iterative method based on a numerical integration by using the Coiflets orthogonal wavelets for a nonlinear fractional differential equation. As an example, an iterative method which can solve the singular nonlinear fractional Riccati equation is presented.

In the paper of Q.-H. Yao and X. Pan the dispersion behavior of leaking hydrogen in a partially open space is simulated by a balancing domain decomposition method. Numerical tests are validated by comparing with the experimental data and confirm the theoretical results.

Finally, Y.-H. Zhao and X.-L. Li analyze the third-order three-point boundary value problem

$$
\begin{array}{ll}
u^{\prime \prime \prime}(t)=f(t, u(t)), & t \in[0,1] \\
u^{\prime}(0)=u(1)=0, & u^{\prime \prime}(\eta)+\alpha u(0)=0,
\end{array}
$$

where $\alpha \in[0,2)$ and $\eta \in[2 / 3,1)$. They obtain the existence of monotone positive solution under some suitable conditions on $f$ by applying iterative method.

\section{Acknowledgments}

The guest editors would like to express their gratitude to all of those who submitted papers for publication and to the many reviewers whose reports were essential for them. Alicia Cordero and Juan R. Torregrosa were partially supported by Ministerio de Ciencia y Tecnología MTM2011-28636-C02-02.

Juan R. Torregrosa Ioannis K. Argyros

Changbum Chun Alicia Cordero Fazlollah Soleymani 


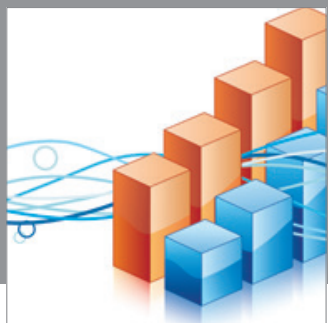

Advances in

Operations Research

mansans

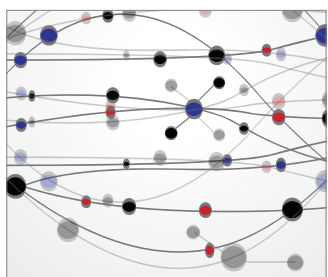

The Scientific World Journal
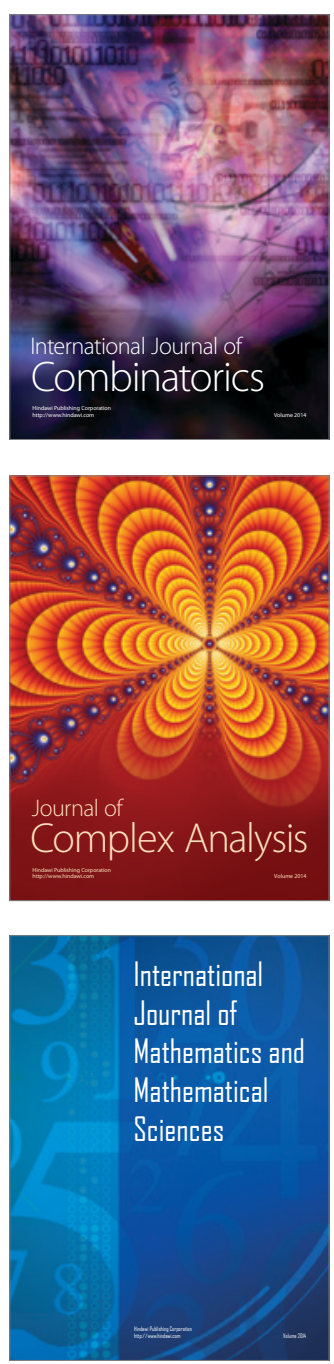
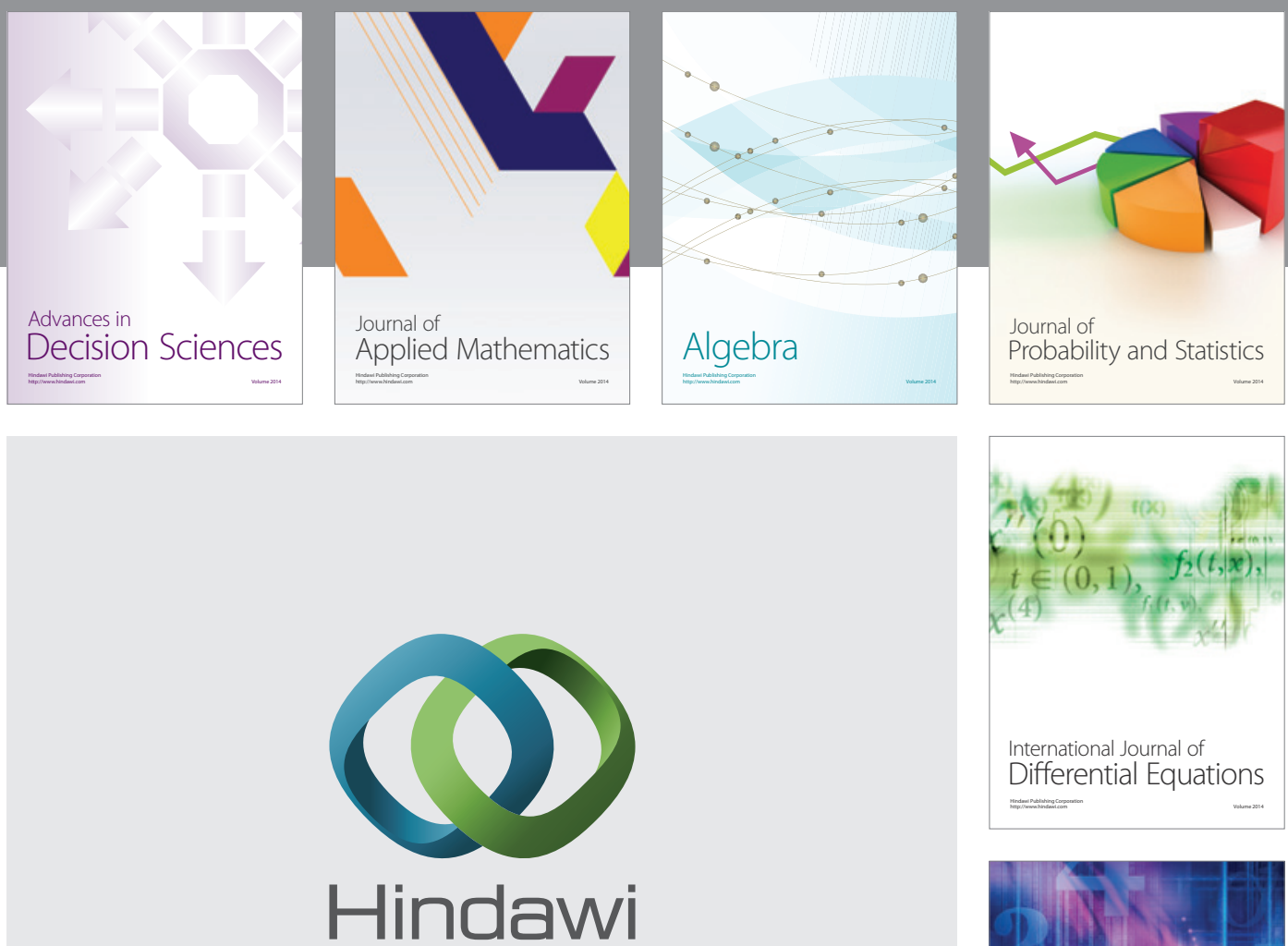

Submit your manuscripts at http://www.hindawi.com
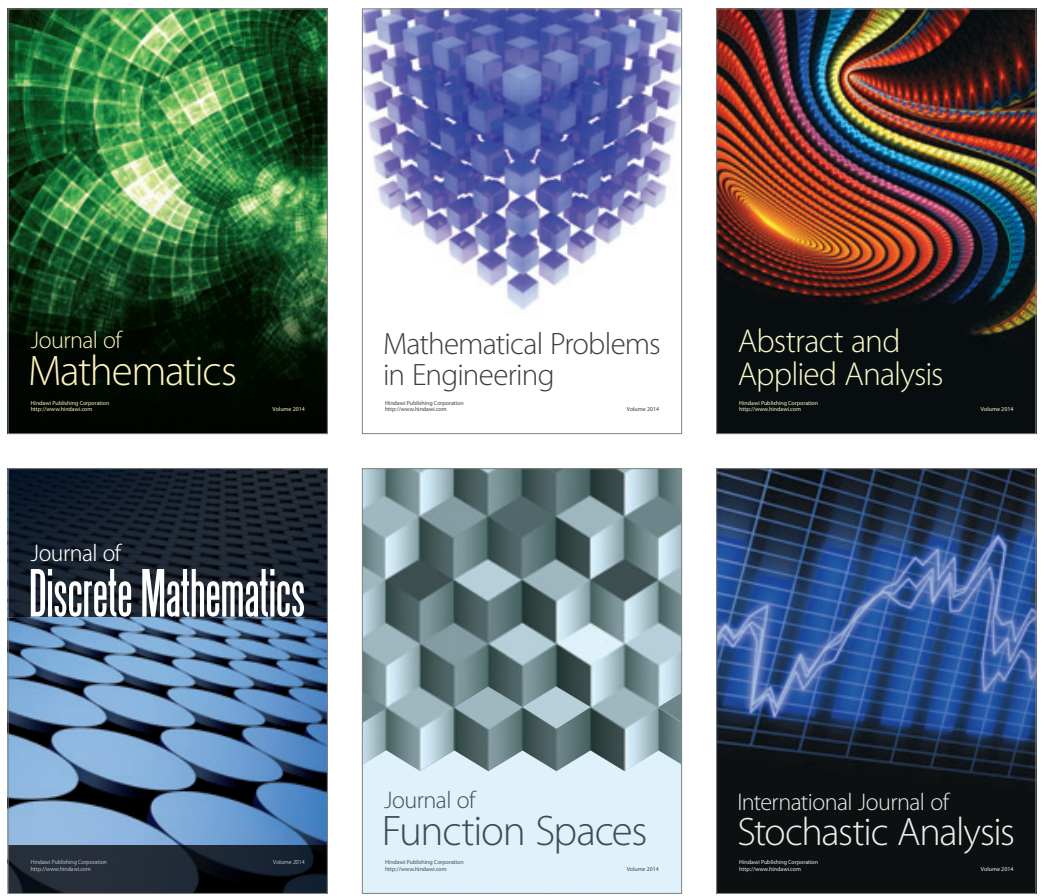

Journal of

Function Spaces

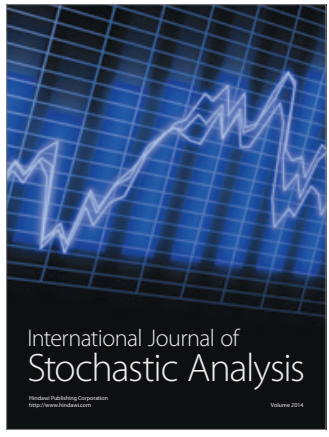

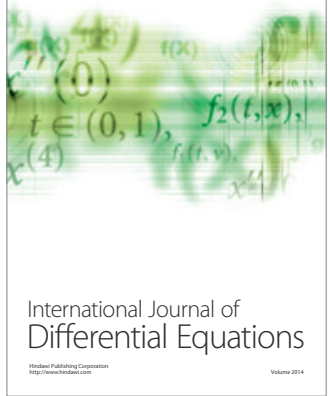
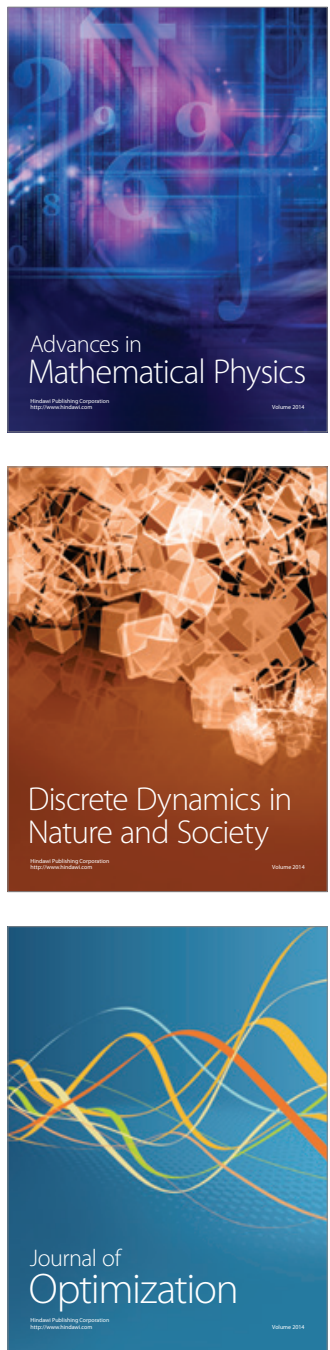